\title{
On Vertex Covering Transversal Domination Number of Regular Graphs
}

\author{
R. Vasanthi ${ }^{1}$ and K. Subramanian ${ }^{2}$ \\ ${ }^{1}$ Department of Mathematics, Alagappa Chettiar College of Engineering and Technology, Karaikudi, \\ Tamil Nadu 630 004, India \\ ${ }^{2}$ Department of Mathematics, Alagappa Government Arts College, Karaikudi, Tamil Nadu 630 003, India
}

Correspondence should be addressed to R. Vasanthi; vasanthi2014accet@gmail.com

Received 19 December 2015; Revised 22 February 2016; Accepted 6 March 2016

Academic Editor: Kinkar Ch Das

Copyright (c) 2016 R. Vasanthi and K. Subramanian. This is an open access article distributed under the Creative Commons Attribution License, which permits unrestricted use, distribution, and reproduction in any medium, provided the original work is properly cited.

A simple graph $G=(V, E)$ is said to be $r$-regular if each vertex of $G$ is of degree $r$. The vertex covering transversal domination number $\gamma_{\mathrm{vct}}(G)$ is the minimum cardinality among all vertex covering transversal dominating sets of $G$. In this paper, we analyse this parameter on different kinds of regular graphs especially for $Q_{n}$ and $H_{3, n}$. Also we provide an upper bound for $\gamma_{\mathrm{vct}}$ of a connected cubic graph of order $n \geq 8$. Then we try to provide a more stronger relationship between $\gamma$ and $\gamma_{\mathrm{vct}}$.

\section{Introduction}

Hamid [1] introduced independent transversal domination in graphs. It was defined using maximum independent set in a graph. Vasanthi and Subramanian [2] introduced vertex covering transversal domination in graphs using minimum vertex covering set in a graph. The vertex covering transversal domination number of some standard graphs such as $K_{n}$, $K_{m, n}, P_{n}, C_{n}$, and $W_{n}$ and trees is dealt with in paper [2]. Bounds of $\gamma_{\mathrm{vct}}$ are also established through various parameters in [2]. Lam et al. [3] worked on independent domination number of regular graphs. In this paper, we investigate our parameter $\gamma_{\mathrm{vct}}$ for regular graphs. Also we try to provide a more stronger relationship between $\gamma$ and $\gamma_{\mathrm{vct}}$.

A simple graph $G=(V, E)$ is said to be $r$-regular if each vertex of $G$ is of degree $r$. A set $I \subseteq V$ of vertices in $G$ is called an independent set if no two vertices in $I$ are adjacent. Also $I$ is said to be a maximum independent set if there is no other independent set $I^{\prime}$ such that $\left|I^{\prime}\right|>|I|$. The cardinality of a maximum independent set is called the independence number and is denoted by $\beta_{0}(G)$. A set $C \subseteq V$ of vertices in $G$ is called a vertex covering set (or simply covering set) if every edge of $G$ is incident to at least one vertex in $C$. Also $C$ is said to be a minimum vertex covering set if there is no other vertex covering set $C^{\prime}$ such that $\left|C^{\prime}\right|<|C|$. The cardinality of a minimum vertex covering set is called the vertex covering number and is denoted by $\alpha_{0}(G)$. A set $D \subseteq V$ of vertices in the graph $G$ is called a dominating set if every vertex in $V-D$ is adjacent to a vertex in $D$. A dominating set which intersects every minimum vertex covering set in $G$ is called a vertex covering transversal dominating set. The minimum cardinality of a vertex covering transversal dominating set is called vertex covering transversal domination number of $G$ and is denoted by $\gamma_{\mathrm{vct}}(G)$.

The parameter independent domination number $i(G)$ was introduced by Cockanye and Hedetniemi in [4]. The independent domination number $i(G)$ is the minimum cardinality among all independent dominating sets of $G$. An independent set is dominating if and only if it is maximal. So $i(G)$ is the minimum cardinality of a maximal independent set in $G$. In paper [3], the following theorem which gives the upper bound for independent domination number of a connected cubic graph has been proved.

Theorem 1. If $G$ is a connected cubic graph of order $n$ where $n \geq 8$, then $i(G) \leq 2 n / 5$. 


\section{Notations}

We use the following notations throughout the paper:

$\alpha_{0}$-set to denote minimum vertex covering set,

$\beta_{0}$-set to denote maximum independent set,

$\gamma$-set to denote a dominating set of minimum cardinality,

$\gamma_{\mathrm{vct}}$-set to denote a vertex covering transversal dominating set of minimum cardinality,

$\gamma(G)$ to denote domination number of $G$,

$\gamma_{\mathrm{vct}}(G)$ to denote vertex covering transversal domination number of $G$,

$i(G)$ to denote independent domination number of $G$,

$O(G)$ to denote the order of $G$,

$\operatorname{deg}_{G}(u)$ to denote the degree of a vertex $u$ in $G$.

\section{3. $\gamma_{\mathrm{vct}}$ for Regular Graphs}

Here, we provide the vertex covering transversal domination number of some standard regular graphs such as complete graphs, complete bipartite regular graphs, cycles, and hypercube $Q_{n}$. We also establish $\gamma_{\text {vct }}$ for certain family of regular graphs defined in [3].

Example 2. $K_{n}$ is a $(n-1)$-regular graph and $\gamma_{\mathrm{vct}}\left(K_{n}\right)=2$ for $n \geq 2$.

Example 3. $C_{n}$ is a 2-regular graph of order $n \geq 3$ and

$$
\gamma_{\mathrm{vct}}\left(C_{n}\right)= \begin{cases}2 & \text { if } n=3,4 \\ 3 & \text { if } n=5 \\ \left\lceil\frac{n}{3}\right] & \text { otherwise. }\end{cases}
$$

Example 4. $K_{n, n}$ is a complete bipartite $n$-regular graph and $\gamma_{\mathrm{vct}}\left(K_{n, n}\right)=2$.

The following theorem provides the vertex covering transversal domination number of $n$-dimensional cube or hypercube $Q_{n}$ defined in [5].

Theorem 5. If $Q_{n}$ is a hypercube containing $2^{n}$ vertices which is n-regular, then

$$
\gamma_{v c t}\left(Q_{n}\right)= \begin{cases}2 & \text { if } n=2 \\ 2^{n-2} & \text { if } n \geq 3 .\end{cases}
$$

Proof. The $n$-dimensional cube or hypercube $Q_{n}$ contains $2^{n}$ vertices and is $n$-regular. Each vertex in $Q_{n}$ is represented by a $n$-tuple with 0's and 1's. Two vertices in $Q_{n}$ are adjacent if and only if the $n$-tuples differ in exactly one position. Also any $v \in Q_{n}$ is the $n$-tuple binary number and its complement $v^{c}$ is also an $n$-tuple binary number obtained by replacing 0 by 1 and 1 by 0 in $v$. The weight of a 0,1 vertex is the number of 1 's occurring in it. There are exactly $2^{n-1}$ vertices of odd weight

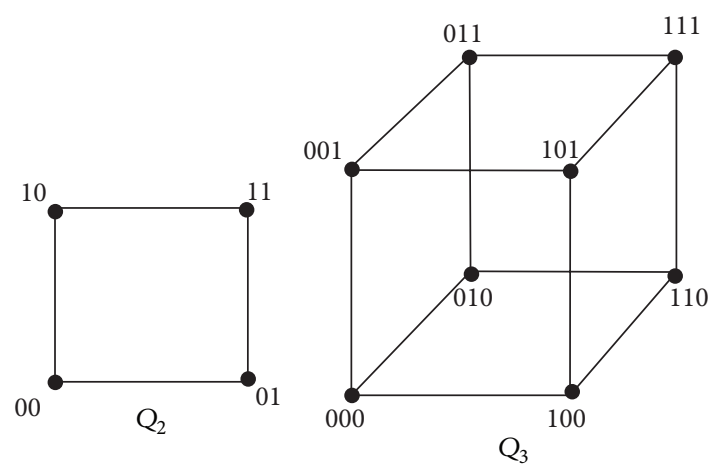

Figure 1

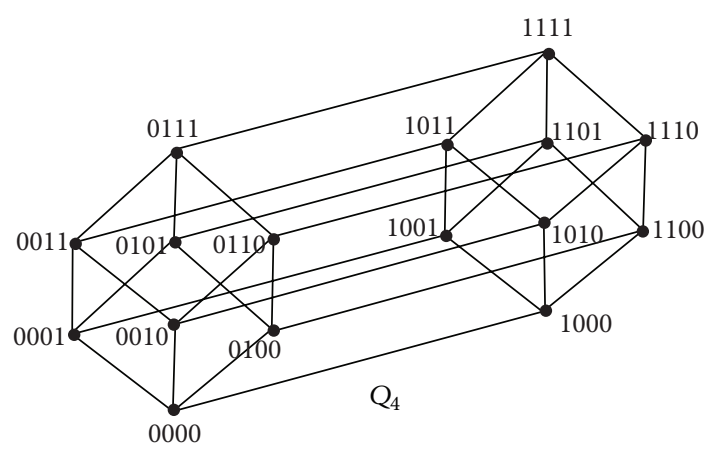

Figure 2

and $2^{n-1}$ vertices of even weight. Each edge of $Q_{n}$ consists of a vertex of even weight and a vertex of odd weight. The vertices of even weight form an independent set and so do the vertices of odd weight. Therefore $Q_{n}$ is bipartite with bipartitions $S_{1}$ and $S_{2}$ where $S_{1}$ is the set of all $n$-tuples of even weight and $S_{2}$ is the set of all $n$-tuples of odd weight with $\left|S_{1}\right|=\left|S_{2}\right|=2^{n-1}$.

Also $S_{1}$ and $S_{2}$ are the only $\beta_{0}$-sets of $Q_{n}$. Since they are complements of each other, $S_{1}$ and $S_{2}$ are the only $\alpha_{0}$-sets of $Q_{n}$.

For $n=2, Q_{2}$ is as shown in Figure 1. Obviously, $S_{1}=$ $\{00,11\}$ and $S_{2}=\{10,01\}$ are $\alpha_{0}$-sets of $Q_{2}$. Then $D=\{00,10\}$ is a $\gamma_{\mathrm{vct}}$-set of $Q_{2}$ and so $\gamma_{\mathrm{vct}}\left(Q_{2}\right)=2$.

Now suppose $n \geq 3$.

For $n=3, Q_{3}$ is the hypercube on 8 vertices which is 3-regular and is represented as in Figure 1. The only two $\alpha_{0}$ - sets of $Q_{3}$ are $S_{1}=\{000,011,110,101\}$ and $S_{2}=$ $\{001,010,100,111\}$. Then $\gamma_{\mathrm{vct}}\left(Q_{3}\right)=2$ since every twoelement set of the form $\left\{v, v^{c}\right\}$ where $v \in S_{1}$ and $v^{c} \in S_{2}$ is a $\gamma_{\mathrm{vct}}$-set.

If $n=4$, the hypercube $Q_{4}$ contains $2^{4}$ vertices and is 4 regular as shown in Figure 2. $Q_{4}$ is bipartite with bipartitions $S_{1}=\{0000,0011,0110,1100,0101,1010,1001,1111\}$ and $S_{2}=\{0001,0010,0100,1000,0111,1110,1011,1101\}$. Also $S_{1}$ and $S_{2}$ are the only $\alpha_{0}$-sets of $Q_{4}$.

Let $D=\{0000,1111,0001,1110\}$. Then $D$ is a dominating set of $Q_{4}$. Clearly it intersects both $S_{1}$ and $S_{2}$. Therefore $D$ is a vertex covering transversal dominating set and so $\gamma_{\mathrm{vct}}\left(Q_{4}\right)=$ 4. Hence it remains to show that $D$ is of minimum cardinality. 


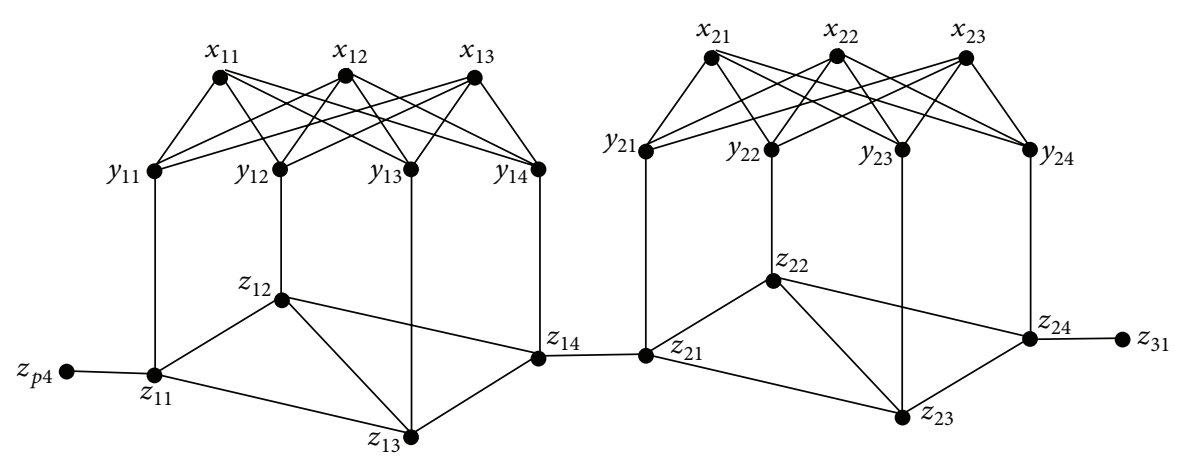

Figure 3

Suppose there exists a vertex covering transversal dominating set $D^{\prime}$ of cardinality less than 4 . It must have at least 2 vertices as it intersects both $S_{1}$ and $S_{2}$. Suppose $\left|D^{\prime}\right|=3$. Since each vertex is of degree 4 , all the three vertices in $D^{\prime}$ may dominate at most 12 vertices. But there are 16 vertices in $Q_{4}$ and so $D^{\prime}$ do not dominate at least 1 vertex. This is a contradiction to the assumption that $D^{\prime}$ is a vertex covering transversal dominating set.

Also any set containing two mutually complementary vertices from $S_{1}$, say, 1100, 0011, and the other two mutually complementary vertices from $S_{2}$, say, 1000, 0111, form a $\gamma_{\text {vct }^{-}}$ set. Thus $S=\left\{v_{1}, v_{1}^{c}, v_{2}, v_{2}^{c}\right\}$ where $v_{1}, v_{1}^{c} \in S_{1}$ and $v_{2}, v_{2}^{c} \in S_{2}$ is a $\gamma_{\mathrm{vct}}$-set of $Q_{4}$.

If $n=5$, the hypercube $Q_{5}$ contains $2^{5}=32$ vertices and the bipartition $S_{1}$ contains $2^{4}$ vertices and $S_{2}$ contains $2^{4}$ vertices. Let $S=\left\{v_{1}, v_{1}^{c}, v_{2}, v_{2}^{c}, v_{3}, v_{3}^{c}, v_{4}, v_{4}^{c}\right\}$ where $v_{1}, v_{2}, v_{3}, v_{4} \in$ $S_{1}$ and $v_{1}^{c}, v_{2}^{c}, v_{3}^{c}, v_{4}^{c} \in S_{2}$. Then $S$ is a $\gamma$-set which intersects both $S_{1}$ and $S_{2}$. Hence $\gamma_{\mathrm{vct}}\left(Q_{5}\right)=2^{3}$.

Thus in general, $S=\left\{v_{1}, v_{1}^{c}, v_{2}, v_{2}^{c}, \ldots, v_{2^{n-3}-1}, v_{2^{n-3}-1}^{c}, v_{2^{n-3}}\right.$, $\left.v_{2^{n-3}}^{c}\right\}$ is a $\gamma_{\mathrm{vct}}$-set of $Q_{n}$. In particular, if $n$ is odd, $v_{1}, v_{2}, \ldots, v_{2^{n-3}} \in S_{1}$ and $v_{1}^{c}, v_{2}^{c}, \ldots, v_{2^{n-3}}^{c} \in S_{2}$. If $n$ is even, $v_{1}, v_{1}^{c}, v_{2}, v_{2}^{c}, \ldots, v_{2^{n-4}}, v_{2^{n-4}}^{c} \in S_{1}$ and $v_{2^{n-4}+1}, v_{2^{n-4}+1}^{c}, \ldots, v_{2^{n-3}}$, $v_{2^{n-3}}^{c} \in S_{2}$. Hence $\gamma_{\mathrm{vct}}\left(Q_{n}\right)=2^{n-2}$.

Theorem 6. If $G$ is a connected regular graph of degree $n-2$ and $O(G)=n$, then $\gamma_{v c t}(G)=2$.

Proof. Choose any vertex $u \in V(G)$. Then $\operatorname{deg}_{G}(u)=n-2$; that is, $u$ is adjacent to $n-2$ vertices in $G$. Then there remains exactly one vertex, say, $v$, which is not adjacent to $u$. Therefore $S=\{u, v\}$ is an independent set of $G$. Also $v$ is adjacent to $n-2$ vertices in $G$ except $u$. Hence no other vertex may be included in $S$. Therefore $S$ is a maximum independent set of $G$.

Now let $w \in V-S$. Then $w$ is adjacent to both $u$ and $v$. Since $u$ dominates every vertex in $G$ except $v$, and $w$ dominates $n-2$ vertices including $v$, it is obvious that $D=$ $\{u, w\}$ is a dominating set which intersects every minimum vertex covering set of $G$. Also $D$ is of minimum cardinality in $G$. Hence $\gamma_{\mathrm{vct}}(G)=2$.

It is noted that $\{v, w\}$ is also a $\gamma_{\mathrm{vct}}{ }^{- \text {set. }}$

Remark 7. In the above theorem, $n$ should be even. For otherwise, if $n$ is odd, then $n-2$ is odd which is impossible as the number of vertices of odd degree in a graph is even.
Lemma 8. Given positive integers $p \geq 2$ and $q \geq 3$, let $G(p, q)$ be the family of graphs such that $V=\bigcup_{i=1}^{p}\left(X_{i} \cup Y_{i} \cup Z_{i}\right)$ and $E=E_{1} \cup E_{2} \cup E_{3} \cup E_{4}$ with

$$
\begin{aligned}
& X_{i}=\left\{x_{i 1}, x_{i 2}, \ldots, x_{i(q-1)}\right\}, Y_{i}=\left\{y_{i 1}, y_{i 2}, \ldots, y_{i q}\right\}, Z_{i}= \\
& \left\{z_{i 1}, z_{i 2}, \ldots, z_{i q}\right\}, \\
& E_{1}=\bigcup_{i=1}^{p}\left\{x_{i k} y_{i l} ; 1 \leq k \leq q-1,1 \leq l \leq q\right\}, \\
& E_{2}=\bigcup_{i=1}^{p}\left\{y_{i k} z_{i k} ; 1 \leq k \leq q\right\}, \\
& E_{3}=\bigcup_{i=1}^{p}\left\{z_{i k} z_{i l} ; 1 \leq k, l \leq q, k \neq l\right\}-\left\{z_{i 1} z_{i q}\right\}, \\
& E_{4}=\left\{z_{i q} z_{(i+1) 1} ; 1 \leq i \leq p-1\right\} \cup\left\{z_{p q} z_{11}\right\} .
\end{aligned}
$$

Then

(i) $|V(G)|=p(3 q-1)$,

(ii) $G(p, q)$ is connected and $q$-regular,

(iii) $\gamma_{v c t}(G(p, q))=4 p$.

Proof. $G(p, q)$ contains $p$ subgraphs which we shall call blocks each containing $3 q-1$ vertices and isomorphic to each other. By the edge set $E_{4}$, we observe that they are connected to each other.

Thus (i) and (ii) are obvious.

For $q=4$, two connected blocks of $G(p, 4)$ each consisting of 11 vertices are as shown in Figure 3.

Now $I=\bigcup_{i=1}^{p} Y_{i}$ is a maximum independent set of $G(p, q)$. Then its complement $S=V-I=\bigcup_{i=1}^{p}\left(X_{i} \cup Z_{i}\right)$ is a minimum vertex covering set of $G(p, q)$.

Also $I_{j}=\bigcup_{i=1}^{p}\left(Y_{i}-\left\{y_{i j}\right\}\right) \cup\left\{z_{i j}\right\}$ and $J_{j}=\bigcup_{i=1}^{p}\left(X_{i}\right) \cup\left\{z_{i j}\right\}$, $1 \leq j \leq q$ are maximum independent sets in $G(p, q)$. Let $S_{j}$ and $T_{j}$ be the complement of each $I_{j}$ and $J_{j}$. Then $S_{j}=$ $\bigcup_{i=1}^{p}\left(\left(X_{i} \cup Z_{i}\right)-\left\{z_{i j}\right\} \cup\left\{y_{i j}\right\}\right)$ and $T_{j}=\bigcup_{i=1}^{p}\left(\left(Y_{i} \cup Z_{i}\right)-\left\{z_{i j}\right\}\right)$, $1 \leq j \leq q$ are minimum vertex covering sets in $G(p, q)$. Now the subgraph $G_{i}$ induced by $X_{i} \cup Y_{i}$ in each block is a complete bipartite graph $K_{q-1, q}$.

Since $\gamma_{\mathrm{vct}}\left(K_{m, n}\right)=2$ if $m, n>1$, we have $\gamma_{\mathrm{vct}}\left(K_{q-1, q}\right)=2$. Also each $\left\{x_{i j}, y_{i k}\right\}$ for $1 \leq j \leq q-1$ and $1 \leq k \leq q$ is a vertex covering transversal dominating set of $G_{i}$. Then $\left\{x_{i j}, y_{i k}, z_{i 1}, z_{i q}\right\}, 1 \leq j \leq q-1$ and $1 \leq k \leq q$, is a dominating set for each block. Therefore $D_{j k}=\bigcup_{i=1}^{p}\left\{x_{i j}, y_{i k}, z_{i 1}, z_{i q}\right\}$, 


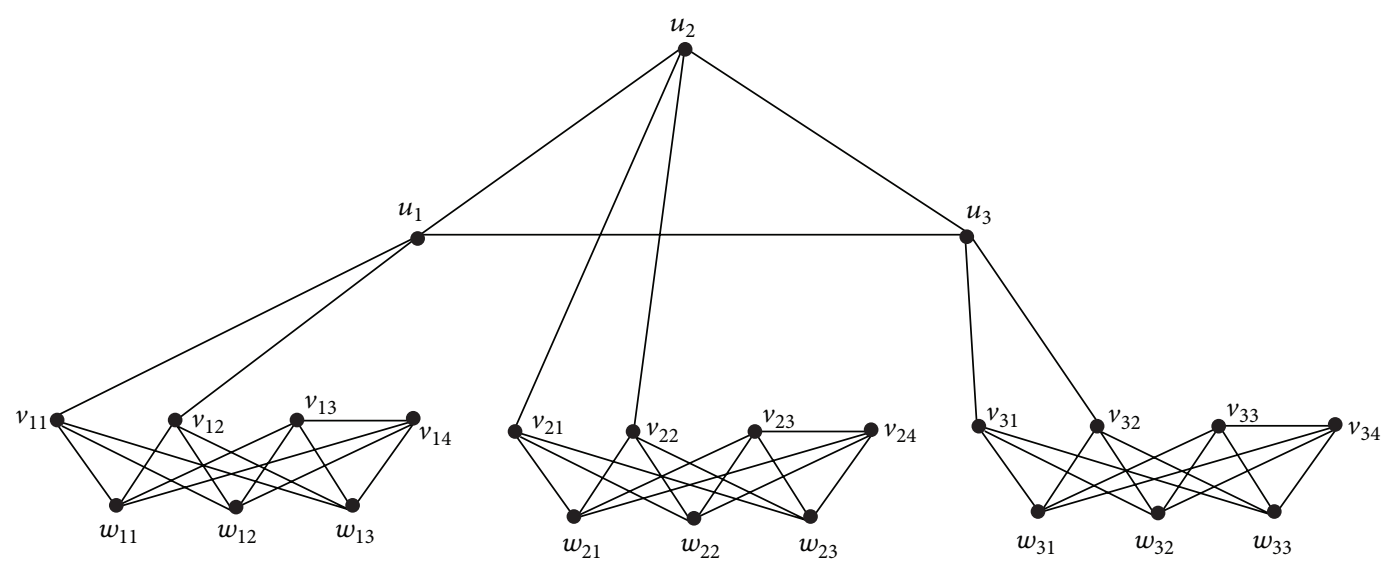

FIGURE 4

$1 \leq j \leq q-1$ and $1 \leq k \leq q$, is a $\gamma$-set which intersects the only $\alpha_{0}$-sets $S, S_{j}$ and $T_{j}$ of $G(p, q)$ for each $j$.

Hence $\gamma_{\mathrm{vct}}(G(p, q))=4 p$.

Theorem 9. If $q \geq 3$, then there exists a connected $q$-regular graph with $\gamma_{v c t}(G) \geq\lceil 4 n / 3 q\rceil$ where $n$ is the order of $G$.

Proof. Let $G=G(p, q)$ be defined as in Lemma 8. Then $\gamma_{\mathrm{vct}}(G) / n=4 /(3 q-1) \geq 4 / 3 q$. Thus $\gamma_{\mathrm{vct}}(G) \geq 4 n / 3 q$.

Lemma 10. Given positive integers $p \geq 1$ and $q \geq 2$, let $G^{*}(p, q)$ be the graph $(V, E)$ with $V=U \cup\left[\bigcup_{i=1}^{2 p+1}\left(V_{i} \cup W_{i}\right)\right]$ and $E=E_{1} \cup E_{2} \cup E_{3} \cup E_{4}$ with

$$
\begin{aligned}
& U=\left\{u_{1}, u_{2}, \ldots, u_{2 p+1}\right\}, V_{i}=\left\{v_{i 1}, v_{i 2}, \ldots, v_{i(q+2 p)}\right\}, \\
& W_{i}=\left\{w_{i 1}, w_{i 2}, \ldots, w_{i(q+2 p-1)}\right\}, \\
& E_{1}=\left\{u_{j} u_{k} ; 1 \leq j<k \leq 2 p+1\right\}, \\
& E_{2}=\bigcup_{i=1}^{2 p+1}\left\{u_{i} v_{i k} ; 1 \leq k \leq q\right\}, \\
& E_{3}=\bigcup_{i=1}^{2 p+1}\left\{v_{i(q+2 k-1)} v_{i(q+2 k)} ; 1 \leq k \leq p\right\}, \\
& E_{4}=\bigcup_{i=1}^{2 p+1}\left\{v_{i k} w_{i l} ; 1 \leq k \leq q+2 p, 1 \leq l \leq q+2 p-1\right\} .
\end{aligned}
$$

Then

(i) $|V(G)|=2(2 p+1)(q+2 p)$,

(ii) $G^{*}(p, q)$ is connected and $(q+2 p)$-regular,

(iii) $\gamma_{v c t}\left(G^{*}(p, q)\right)=2(2 p+1)$.

Proof. (i) and (ii) are obvious. If $p=1$ and $q=2$, the graph $G^{*}(1,2)$ is as shown in Figure 4.

It is clear that each $I_{j}=\left[\bigcup_{i=1}^{2 p+1}\left(W_{i}\right)\right] \cup\left\{u_{j}\right\}, j=1$ to $2 p+1$, is a maximum independent set in $G^{*}(p, q)$. Therefore its complement $J_{j}=V-I_{j}=\left[\bigcup_{i=1}^{2 p+1}\left(V_{i}\right)\right] \cup\left[U-\left\{u_{j}\right\}\right]$, $j=1$ to $2 p+1$, is an $\alpha_{0}$ set in $G^{*}(p, q)$. Further $J_{1}, J_{2}, \ldots, J_{2 p}$ and $J_{2 p+1}$ are the only $\alpha_{0}$-sets of $G^{*}(p, q)$. Now each $S_{j k}=$ $\bigcup_{i=1}^{2 p+1}\left\{v_{i k}, w_{i j}\right\}, j=1$ to $q+2 p-1, k=1$ to $q$ is a dominating set intersecting $J_{1}, J_{2}, \ldots, J_{2 p}$ and $J_{2 p+1}$ and also of minimum cardinality $2(2 p+1)$.

Hence $\gamma_{\mathrm{vct}}\left(G^{*}(p, q)\right)=2(2 p+1)$.
Theorem 11. For everyr $\geq 4$, there exists a connected $r$-regular graph $G$ of order $n$ such that $\gamma_{v c t}(G)=n / r$.

Proof. Let $G=G^{*}(p, q)$ be defined as in Lemma 10. Then $G$ is a connected $r$-regular graph with $r=q+2 p$. Also $\gamma_{\mathrm{vct}}(G) / n=$ $1 /(q+2 p)$

$$
\text { Thus } \gamma_{\mathrm{vct}}(G)=n / r \text {. }
$$

Remark 12. Theorems 9 and 11 hold good if $\gamma_{\mathrm{vct}}$ is replaced by $\gamma$

\section{4. $\gamma_{\mathrm{vct}}$ for Regular Cubic Graphs}

In this section, we provide the vertex covering transversal domination number of some regular cubic graphs especially Harary graph $H_{3, n}$ defined in [6]. We also obtain an upper bound for the vertex covering transversal domination number of a connected cubic graph.

Example 13. Consider the triangular prism graph $Y_{3}$ shown in Figure 5. It is a regular cubic graph.

$Y_{3}$ has 6 vertices and 9 edges. Assume that the graph $Y_{3}$ is labelled as shown in the diagram. It is clear that $\left\{u_{i \bmod 3}, v_{(i+1) \bmod 3}\right\}$ and $\left\{u_{i \bmod 3}, v_{(i+2) \bmod 3}\right\}$ for $i=0,1,2$ are $\beta_{0}$-sets of $Y_{3}$. Then their complements $C_{i}=\left\{u_{(i+1) \bmod 3}, u_{(i+2) \bmod 3}, v_{i \bmod 3}, v_{(i+2) \bmod 3}\right\}$ and $S_{i}=$ $\left\{u_{(i+1) \bmod 3}, u_{(i+2) \bmod 3}, v_{i \bmod 3}, v_{(i+1) \bmod 3}\right\}$ for $i=0,1,2$ are $\alpha_{0}$-sets of $Y_{3}$. Now each $D_{i}=\left\{u_{i \bmod 3}, v_{i \bmod 3}\right\}, i=0,1,2$ is a $\gamma$-set for $Y_{3}$. Clearly it intersects each $C_{i}$ and $S_{i}$. Therefore $\gamma_{\mathrm{vct}}\left(Y_{3}\right)=2$.

Example 14. Consider Peterson graph which is cubic regular shown in Figure 6.

Assuming that the graph $G$ is labelled as shown in Figure 6 , it is obvious that $I_{i}=\left\{v_{i \bmod 5}, v_{(i+3) \bmod 5}\right.$, $\left.u_{(i+1) \bmod 5}, u_{(i+2) \bmod 5}\right\}, i=0,1,2,3,4$ are $\beta_{0}$-sets of $G$. Then their complements $C_{i}=\left\{v_{(i+1) \bmod 5}, v_{(i+2) \bmod 5}, v_{(i+4) \bmod 5}\right.$, $\left.u_{i \bmod 5}, u_{(i+3) \bmod 5}, u_{(i+4) \bmod 5}\right\}, i=0,1,2,3,4$ are $\alpha_{0}$-sets 


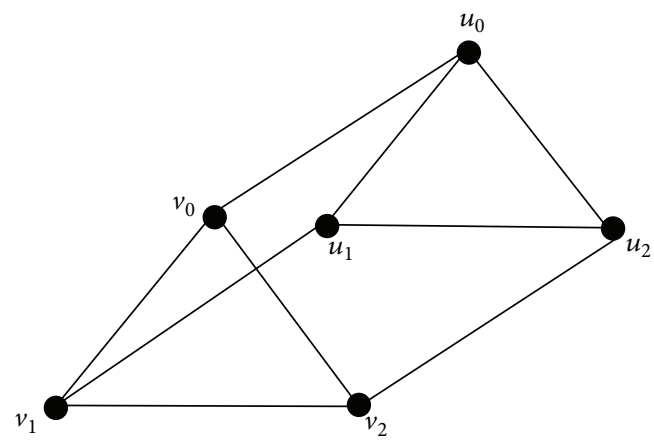

FIGURE 5

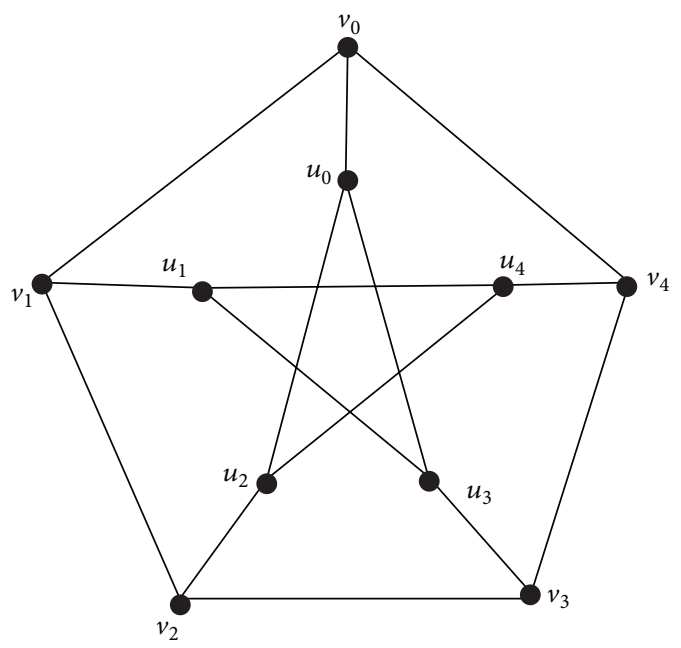

FIGURE 6

of $G$. Now $S_{i}=\left\{v_{i \bmod 5}, u_{(i+2) \bmod 5}, u_{(i+3) \bmod 5}\right\}$ are $\gamma$-sets intersecting each $C_{i}$. Hence $\gamma_{\mathrm{vct}}(G)=3$.

Note that $S_{i}=\left\{v_{i \bmod 5}, v_{(i+3) \bmod 5}, u_{(i+4) \bmod 5}\right\}$ are also $\gamma_{\text {vct }}$-sets in $G$.

Theorem 15. If $H_{3, n}$ is a Harary graph with $n \geq 6$, then $\gamma_{v c t}\left(H_{3, n}\right)=\lfloor(n+1) / 3\rfloor$.

Proof. $H_{3, n}$ is a 3-regular graph and so $n$ is even. By the definition of $H_{3, n}$, every vertex $v_{i} \in H_{3, n}$ is adjacent to the vertices $v_{i+1}, v_{i-1}$, and $v_{i+k}$ where $n=2 k$.

Let $V\left(H_{3, n}\right)=\left\{v_{0}, v_{1}, v_{2}, \ldots, v_{n-1}\right\}$. The graphs $H_{3,10}$ and $H_{3,12}$ are shown in Figure 7.

Case 1. Suppose $n=2 k$ where $k$ is odd.

Then $C_{1}=\left\{v_{0}, v_{2}, v_{4}, \ldots, v_{n-2}\right\}$ and $C_{2}=\left\{v_{1}, v_{3}, v_{5}, \ldots\right.$, $\left.v_{n-1}\right\}$ are the only $\alpha_{0}$-sets of $H_{3, n}$.

Subcase 1. Let $n \equiv 0(\bmod 3)$.

Then $S=\left\{v_{0}, v_{3}, v_{6}, \ldots, v_{n-3}\right\}$ is a $\gamma$-set which intersects $C_{1}$ and $C_{2}$ and $|S|=n / 3$.

Subcase 2. Suppose $n \equiv 1(\bmod 3)$.
Then $S=\left\{v_{0}, v_{3}, v_{6}, \ldots, v_{n-4}\right\}$ is a $\gamma$-set which intersects $C_{1}$ and $C_{2}$ and $|S|=(n-1) / 3$.

Subcase 3. Suppose $n \equiv 2(\bmod 3)$.

Then $S=\left\{v_{0}, v_{3}, v_{6}, \ldots, v_{n-2}\right\}$ is a $\gamma$-set which intersects $C_{1}$ and $C_{2}$ with $|S|=(n+1) / 3$.

Thus in all the subcases of Case $1, \gamma_{\mathrm{vct}}\left(H_{3, n}\right)=\lfloor(n+1) / 3\rfloor$.

Case 2. Suppose $n=2 k$ where $k$ is even.

Then $I_{i}=\left\{v_{i \bmod n}, v_{(i+2) \bmod n}, \ldots, v_{(i+k-2) \bmod n}\right.$, $\left.v_{(i+k+1) \bmod n}, v_{(i+k+3) \bmod n}, \ldots, v_{(i+n-5) \bmod n}, v_{(i+n-3) \bmod n}\right\}$ is a $\beta_{0}$-set for each $i=0,1,2, \ldots, n-1$.

Therefore $C_{i}=\left\{v_{(i+1) \bmod n}, v_{(i+3) \bmod n}, \ldots, v_{(i+k-1) \bmod n}\right.$, $v_{(i+k) \bmod n}, v_{(i+k+2) \bmod n}, \ldots, v_{(i+n-4) \bmod n}, \quad v_{(i+n-2) \bmod n}$, $\left.v_{(i+n-1) \bmod n}\right\}$ is an $\alpha_{0}$-set for each $i=0,1,2, \ldots, n-1$.

Subcase 1 . Let $n \equiv 0(\bmod 3)$.

Then $S_{i}=\left\{v_{i \bmod n}, v_{(i+3) \bmod n}, v_{(i+6) \bmod n}, \ldots\right.$, $\left.v_{(i+n-3) \bmod n}\right\}$ is a $\gamma$-set which intersects each $C_{i}$ for $i=0,1$, $2, \ldots, n-1$.

Subcase 2. Let $n \equiv 1(\bmod 3)$.

Then $S_{i}=\left\{v_{i \bmod n}, v_{(i+3) \bmod n}, v_{(i+6) \bmod n}, \ldots\right.$, $\left.v_{(i+n-4) \bmod n}\right\}$ is a $\gamma$-set which intersects each $C_{i}$ for $i=0,1$, $2, \ldots, n-1$.

Subcase 3. Let $n \equiv 2(\bmod 3)$.

Then $S_{i}=\left\{v_{i \bmod n}, v_{(i+3) \bmod n}, v_{(i+6) \bmod n}, \ldots\right.$, $\left.v_{(i+n-2) \bmod n}\right\}$ is a $\gamma$-set which intersects each $C_{i}$ for $i=0,1$, $2, \ldots, n-1$.

The $\gamma_{\mathrm{vct}}$-sets mentioned in all the subcases of Case 2 are also of cardinality $\lfloor(n+1) / 3\rfloor$.

Thus $\gamma_{\mathrm{vct}}\left(H_{3, n}\right)=\lfloor(n+1) / 3\rfloor$.

Remark 16. In most of the graphs considered by us, it is observed that $\gamma_{\mathrm{vct}}=\gamma$.

Theorem 17. If $G$ is a connected cubic graph of order $n$ with $n \geq 8$, then $\gamma_{v c t}(G) \leq\lceil 2 n / 5\rceil$.

Proof. Let $I$ be an independent dominating set of cardinality $i(G)$. Then $I$ is a maximal independent set of minimum cardinality. Since $I$ is independent, no two vertices of $I$ are adjacent in $G$. Let $J=V-I$. Then the vertices in $I$ are adjacent only to the vertices in $J$.

Case 1. Suppose $I$ itself is a $\beta_{0}$-set. Then $J$ is an $\alpha_{0}$-set. Let $S=I \cup\{v\}$ where $v \in J$. Then $S$ is a vertex covering transversal dominating set of $G$. Therefore $\gamma_{\mathrm{vct}}(G) \leq i(G)+1$. Hence $\gamma_{\mathrm{vct}}(G) \leq 2 n / 5+1$ (by Theorem 1 proved in [3]).

Case 2. Suppose $I$ is not a $\beta_{0}$-set. But $I$ is a maximal independent dominating set of minimum cardinality. We claim that $I$ intersects every $\alpha_{0}$-set of $G$.

Suppose that $I$ does not intersect an $\alpha_{0}$-set $C$ of $G$. Then $I \subset V-C$ where $V-C$ is a $\beta_{0}$-set of $G$. This is a contradiction to the maximality of $I$.

Hence $I$ itself is a vertex covering transversal dominating set of $G$. Therefore $\gamma_{\mathrm{vct}}(G) \leq 2 n / 5$.

Thus Cases 1 and 2 imply that $\gamma_{\mathrm{vct}}(G) \leq\lceil 2 n / 5\rceil$. 

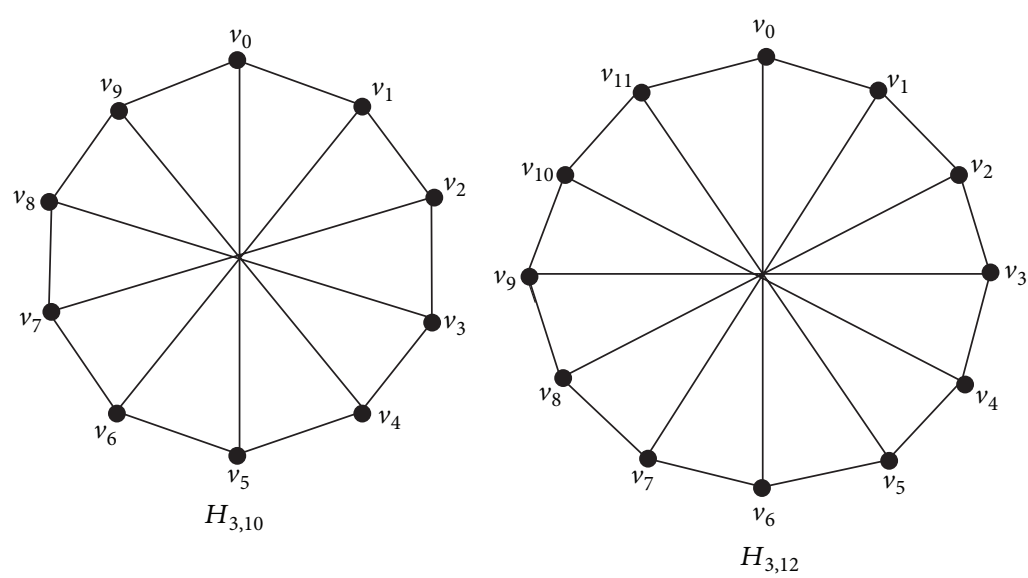

FIGURE 7

\section{Relation between $\gamma(G)$ and $\gamma_{\mathrm{vct}}(G)$}

In this section, we prove a more stronger relationship between $\gamma$ and $\gamma_{\mathrm{vct}}$ than that proved in [2]. In view of the results and theorems dealt with in the previous sections, we try to characterize graphs for which $\gamma=\gamma_{\mathrm{vct}}$ and $\gamma<\gamma_{\mathrm{vct}}$.

Theorem 18. If $G$ is a simple connected graph, then $\gamma_{v c t}(G) \leq$ $\gamma(G)+1$.

Proof. Let $D$ be a minimum dominating set. If $D=V(G)$, then obviously $\gamma_{\mathrm{vct}}(G)=\gamma(G)$. If not, then $D \subset V(G)$ and $V(G)-D \neq \phi$. Let $u \in V(G)-D$. Then $u$ is dominated by some vertex $v$ in $D$. Let $S=D \cup\{u\}$. Since $u v$ is an edge in $G$, either $u$ or $v$ is included in every minimum vertex covering set of $G$. This implies that $S$ intersects every minimum vertex covering set in $G$. Hence $\gamma_{\mathrm{vct}}(G) \leq \gamma(G)+1$.

Theorem 19. Let $G$ be a simple connected graph. If there exists a $\gamma$-set which is not independent, then $\gamma_{v c t}(G)=\gamma(G)$.

Proof. Let $D$ be a minimum dominating set which is not an independent set of $G$. Then at least two vertices, say, $u, v$ in $D$, are adjacent to each other. Therefore $u v$ is an edge in $G$ and hence either $u$ or $v$ lies in every minimum vertex covering set of $G$. So $D$ intersects every $\alpha_{0}$-set of $G$. Therefore $D$ itself is a $\gamma_{\mathrm{vct}}$-set. Hence $\gamma_{\mathrm{vct}}(G)=\gamma(G)$.

Remark 20. The converse is not true. If $\gamma_{\mathrm{vct}}(G)=\gamma(G)$, then there may exist a $\gamma$-set which is independent also. For example, consider $C_{6}$, the cycle on 6 vertices as shown in Figure 8.

Obviously $\left\{v_{1}, v_{3}, v_{5}\right\}$ and $\left\{v_{2}, v_{4}, v_{6}\right\}$ are the only $\alpha_{0}$-sets of $C_{6}$. Also $\left\{v_{1}, v_{4}\right\}$ is a $\gamma$-set which is independent. Further, it is a $\gamma_{\mathrm{vct}}$-set as it intersects both the $\alpha_{0}$-sets of $C_{6}$. Thus there exists a $\gamma$-set which is independent in $C_{6}$ even though $\gamma_{\mathrm{vct}}\left(C_{6}\right)=$ $\gamma\left(C_{6}\right)$.

Remark 21. Now, the obvious question is "If $\gamma_{\mathrm{vct}}(G)=\gamma(G)$, is every $\gamma$-set of $G$ a $\gamma_{\mathrm{vct}}$-set?" The answer is "not always." The $\gamma$-sets and $\gamma_{\mathrm{vct}}$-sets in the graphs $Q_{2}$ and $Y_{3}$ discussed in the previous sections are the best examples for it. So it is noted

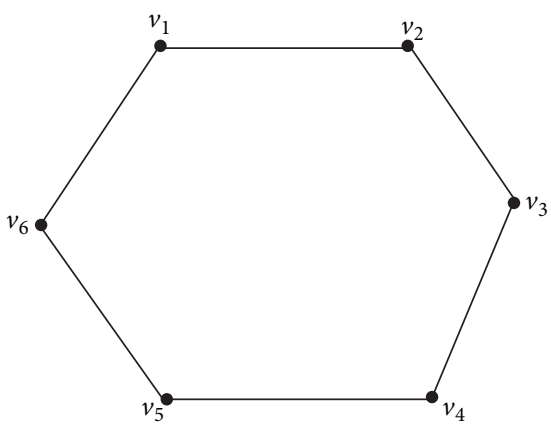

Figure 8

that this happens if there exists a $\gamma$-set which is also a $\beta_{o}$-set. It obviously produces the result that "If $\gamma_{\mathrm{vct}}(G)=\gamma(G)=\beta_{o}(G)$, then there exists at least one $\gamma$-set in $G$ which is not a $\gamma_{\mathrm{vct}}$-set." The next general question is that "What happens if all the $\gamma$ sets of $G$ are $\beta_{0}$-sets?". The following theorem provides the answer to it.

Theorem 22. Let $G$ be a simple connected graph. If every $\gamma$-set of $G$ is a $\beta_{0}$-set, then $\gamma_{v c t}(G)=\gamma(G)+1$.

Proof. Since every $\gamma$-set $D$ of $G$ is a $\beta_{0}$-set, choose a vertex $v$ in its complement. This is possible since $D \neq V(G)$ as $D$ is a $\beta_{0}$-set of a connected graph $G$. Obviously $D$ is not a $\gamma_{\mathrm{vct}}$-set as it does not intersect the $\alpha_{0}$-set $V(G)-D$. Let $S=D \cup\{v\}$. We claim that $S$ intersects every $\alpha_{0}$-set of $G$. Suppose that $S \cap C=$ $\Phi$ for some $\alpha_{0}$-set $C$ in $G$. Then $S \subseteq I$ where $I=V(G)-C$ is a $\beta_{0}$-set. This implies that $\beta_{0}(G)+1 \leq \beta_{0}(G)$ which is a contradiction. Hence $S$ intersects every $\alpha_{0}$-set of $G$. Also $S$ is a $\gamma_{\mathrm{vct}}$-set of $G$ as it contains exactly one vertex more than that of the $\gamma$-set $D$. Thus $\gamma_{\mathrm{vct}}(G)=\gamma(G)+1$.

Remark 23. It is easy to conclude that even though $\gamma_{\mathrm{vct}}=\gamma$, there are graphs in which $\gamma$-sets do not become $\gamma_{\mathrm{vct}}$-sets. This implies that the collection of $\gamma_{\mathrm{vct}}$-sets in such graphs is contained in the collection of $\gamma$-sets. So this may lead to consider $\gamma_{\mathrm{vct}}$-sets in the graphs for which $\gamma_{\mathrm{vct}}=\gamma$ when we are in a situation to select a minimum number of $\gamma$-sets 
in such graphs. This approach may affect a new variation in domination theory.

\section{Competing Interests}

The authors declare that there are no competing interests regarding the publication of this paper.

\section{References}

[1] I. S. Hamid, "Independent transversal domination in graphs," Discussiones Mathematicae. Graph Theory, vol. 32, no. 1, pp. 517, 2012.

[2] R. Vasanthi and K. Subramanian, "Vertex covering transversal domination in graphs," International Journal of Mathematics and Soft Computing, vol. 5, no. 2, pp. 1-7, 2015.

[3] P. C. Lam, W. C. Shiu, and L. Sun, "On independent domination number of regular graphs," Discrete Mathematics, vol. 202, no. 1-3, pp. 135-144, 1999.

[4] E. J. Cockayne and S. T. Hedetniemi, "Towards a theory of domination in graphs," Networks, vol. 7, no. 3, pp. 247-261, 1977.

[5] D. B. West, Introduction to Graph Theory, Prentice-Hall of India Private Limited, New Delhi, India, 2000.

[6] G. Chatrand and P. Zhang, Introduction to Graph Theory, Tata McGraw-Hill Education Private Limited, New Delhi, India, 8th edition, 2012. 


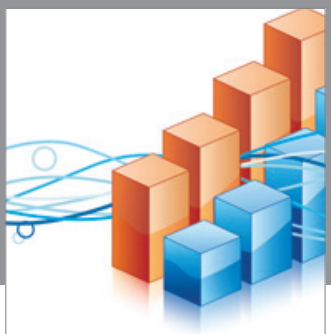

Advances in

Operations Research

vatem alat4

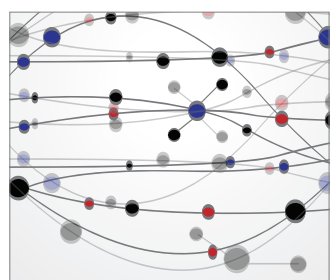

\section{The Scientific} World Journal
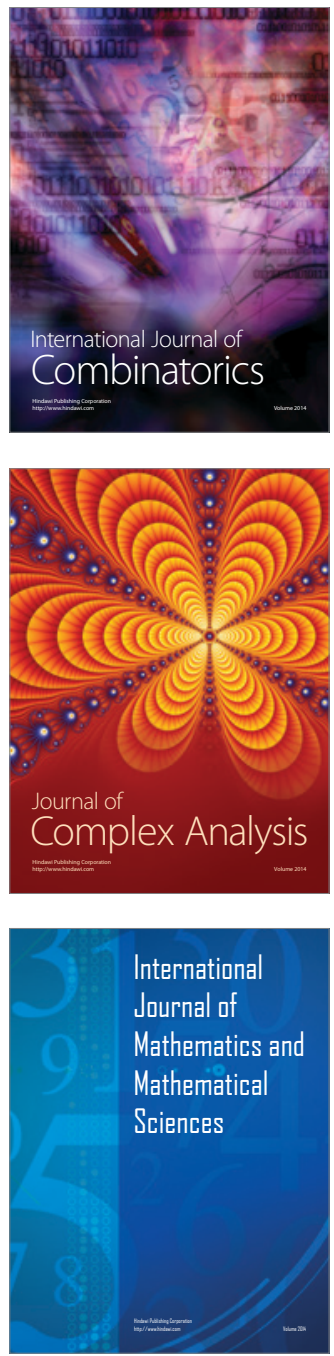
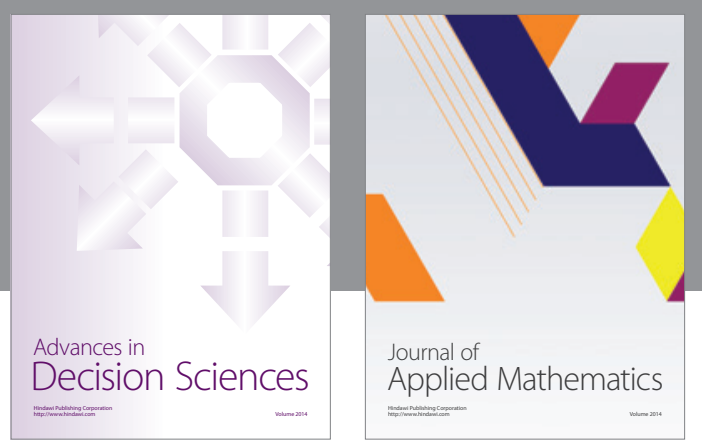

Algebra

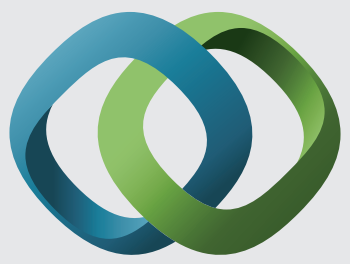

\section{Hindawi}

Submit your manuscripts at

http://www.hindawi.com
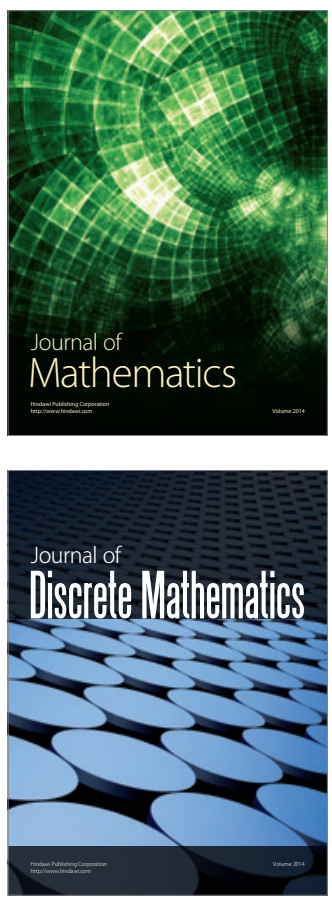

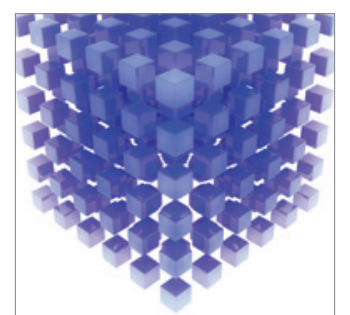

Mathematical Problems in Engineering
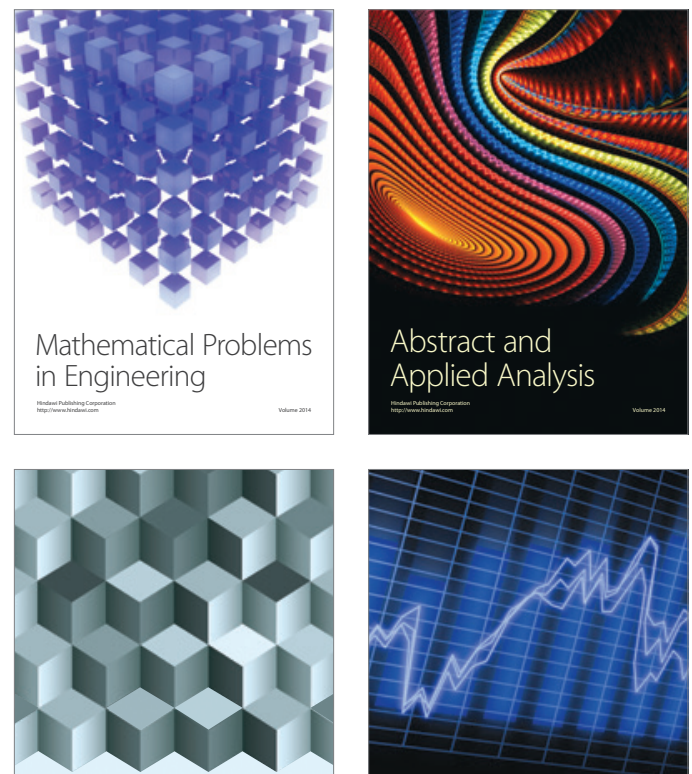

Journal of

Function Spaces

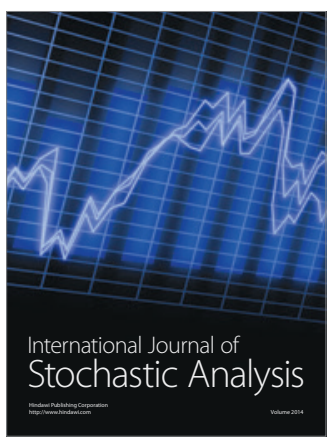

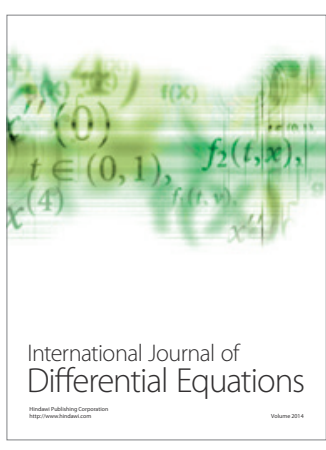
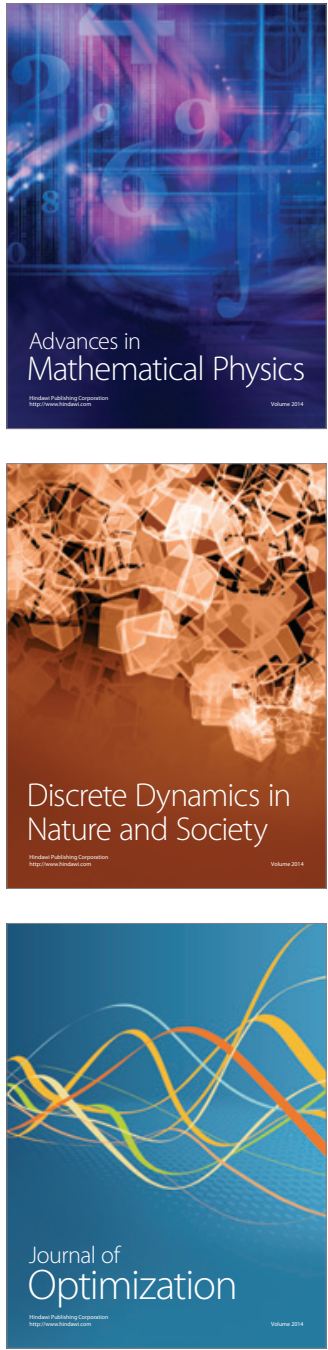